\title{
Courbes d'ébullition en convection forcée Deuxième partie : Effets paramétriques et corrélations
}

\author{
Boiling curves with forced convection \\ Part 2 : Parametrical effects and correlations
}

M. Afify,

D. Gentile,

ENSTA,

Groupe Phénomènes d'Interface, 91120 Palaiseau

\author{
M. Llory \\ EDF \\ Direction des Etudes \\ et recherches \\ 78140 Chatou
}

La première partie de cet article, parue dans le no 6/7-1985, était essentiellement consacrée à une revue bibliographique. Une attention particulière y a été portée sur la notion de courbe d'ébullition (caractéristique d'échange thermique), son intérêt au point de vue industriel, et sur le concept de stabilité thermique.

Dans cette seconde partie, on rappelle les différents dispositifs expérimentaux qui permettent d'atteindre la stabilité thermique. L'analyse et la critique du tracé de courbes d'ébullition et de l'effet de certains paramètres physiques sur leur allure générale sont ensuite effectuées. Enfin, le recensement des principales corrélations existantes est effectué, le choix approprié de certaines d'entre elles permettant de reconstruire numériquement des courbes d'ébullition.

The first part of this article appeared in issue $n^{\circ}$ 6/7-1985, and it was mainly devoted to a bibliographical review. Special attention has been paid to the notion of boiling curve (thermal exchange characteristic). its advantages from the industrial point of view, and the concept of thermal stability.

The second part reviews the various experimental devices which enable thermal stability to be reached. The analysis and the assessment of the trace of boiling curves and the effects of some physical parameters on their general course are then carried out. Finally, the recording of main correlations is carried out, the appropriate choice of some of them enablig boiling curves to be numerically reconstructed.

La première partie [1] était essentiellement consacrée à une revue bibliographique concernant les mécanismes de transferts thermiques en ébullition en convection forcée (écoulements ascendants de fluide le long de surfaces chauffantes circulaires ou annulaires, supposées axisymétriques et monodimensionnelles).

Une attention particulière a été portée sur la notion de courbe d'ébullition (caractéristique d'échange thermique), son intérêt au point de vue industriel, et sur le concept de stabilité thermique. Il a été montré, dans un cas simplifié, que ce critère impose la réalisation de conditions précises pour l'obtention complète de la courbe d'ébullition. Nous ne reviendrons pas sur les conclusions du critère mais renverrons le lecteur à notre première partie et aux références correspondantes.

Nous rappellerons dans cette seconde partie les différents dispositifs expérimentaux qui permettent d'atteindre la stabilité thermique. L'analyse et la critique du tracé de courbes d'ébullition et de l'effet de certains paramètres physiques sur leur allure générale seront effectuées dans un second paragraphe. Enfin le recensement des principales corrélations existantes sera effectué et le choix approprié de certaines d'entre elles permettra de reconstruire numériquement des courbes d'ébullition. 


\section{Rappel des différents dispositifs expérimentaux}

Le principe sur lequel repose la conception de ces dispositifs est une conséquence du critère de stabilité : la condition nécessaire à la réalisation de la stabilité thermique est résumée par l'inégalité

$$
\frac{\partial q_{i}}{\partial \theta}<\frac{\partial q_{i}}{\partial \theta}
$$

La pente de la caractéristique externe en un point de fonctionnement stable, doit être inférieure à la pente, au même point, de la caractéristique interne. Cela implique, en particulier, qu'un système dont le fonctionnement est imposé par le flux ne peut pas être stable dans la zone où $\partial q_{i} / \partial \theta$ est négatif c'est-à-dire celle que l'on appelle généralement la zone d'ébullition de transition.

On distingue dans la littérature spécialisée trois dispositifs permettant d'obtenir la courbe d'ébullition dans sa totalité :

Les deux premiers sont basés sur la réalisation du concept de stabilité thermique

1) les dispositifs statiquement stables,

2) les dispositifs dynamiquement stables,

3) les dispositifs fonctionnant en régime transitoire.

Les deux premiers sont basés sur la réalisation du concept de stabilité thermique.

Ils ont été décrits brièvement dans la première partie et dans les références citées. Nous rappellerons simplement pour chacun d'eux le principe.

\subsection{Dispositifs statiquement stables}

Ils sont conçus pour assurer une température constante en paroi. (Caractéristique externe "verticale", parallèle à l'axe des flux). Ils nécessitent la présence d'un fluide auxiliaire, dit stabilisateur, circulant à contre-courant ou parfois en condensation (température de changement de phase constante). Les mécanismes d'échange entre ce fluide et la paroi, la paroi et le fluide à stabiliser, imposent un fluide stabilisateur de grand coefficient d'échange et une paroi de faible résistance thermique (conductivité thermique élevée et faible épaisseur).

Ainsi que le note Hesse [2] dans ses expériences, la stabilisation sera d'autant meilleure que les vitesses de circulation du fluide stabilisateur seront élevées et la pression du fluide à stabiliser sera basse.

Comme nous l'avons mentionné dans la partie I, un certain nombre de difficultés surgissent dans ce genre de dispositif dont deux essentielles doivent être rappelées :

- la difficulté d'évaluer précisément le flux transmis au fluide à stabiliser, compte tenu des très faibles écarts de température à mesurer entre les fluides chauffant et chauffé;

- le choix restreint du fluide stabilisateur, compte tenu des conditions à remplir. Ainsi les auteurs qui ont mis en œuvre cette méthode ont été conduits à utiliser des fluides comme le sodium ou le mercure, ce qui présente des coûts relativement élevés et des précautions d'utilisation importantes.

En outre, il faut relever dans les principaux articles traitant de cette méthode que peu d'auteurs ont vérifié à postériori si le critère de stabilité était réellement assuré, ce qui rend les résultats obtenus criticables.

\subsection{Dispositifs dynamiquement stables}

Le principe de ces dispositifs est d'assurer la stabilité thermique du phénomène d'ébullition par chauffage électrique direct asservi. Le flux de chaleur n'est pas imposé directement mais par l'intermédiaire d'un signal d'erreur élaboré par comparaison entre une valeur de température mesurée en un point ou sur toute la paroi de la section (valeur moyenne).

C'est le principe d'un asservissement à contre réaction dont le calcul et le fonctionnement ont été décrits dans $[3,4]$. L'étude fonctionnelle et la conception du dispositif font appel aux théories classiques des fonctions de transferts qui nécessitent une hypothèse forte : la linéarisation autour d'un point de fonctionnement stable. La mise en œuvre des critères algébriques et graphiques de stabilité des fonctions de transfert doit permettre d'assurer une stabilité dynamique du système vis-à-vis des perturbations, à condition que celles-ci ne fassent pas sortir le système de l'hypothèse de linéarisation.

$\mathrm{Ce}$ dispositif est d'une utilisation très souple. Cependant sa conception n'est pas aisée car il nécessite une connaissance très précise du mécanisme à stabiliser, en particulier de la dérivée $\partial q_{i} / \partial \theta$ en tout point de la zone instable. Il impose par conséquent une identification du phénomène, c'est-à-dire la connaissance de sa fonction de transfert et de son comportement vis-à-vis des perturbations.

D'autre part, l'influence de la conduction axiale conduisant à des distributions de températures non uniformes le long du tube chauffant complique considérablement la mise en œuvre d'un tel asservissement : on se trouve en fait devant le cas d'un système à paramètres distribués dont l'asservissement ne peut être identique à celui d'un système à paramètres répartis (« lumped parameter system ").

Un travail de recherche tant expérimental que théorique (par voie numérique) est actuellement en cours à I'ENSTA et à l'EDF pour améliorer encore un tel dispositif afin qu'il réponde de façon satisfaisante au problème posé. Les résultats de ces recherches seront prochainement publiés.

\subsection{Dispositifs fonctionnant en régime transitoire}

Leur principe consiste à préchauffer la section d'essais sans liquide à une température supérieure à celle du point de Leidenfrost et à injecter ensuite le fluide. L'enregistrement de la température de refroidissement du tube au cours du temps permet de reconstruire une courbe d'ébullition. Le passage de la fonction $\theta(t)$ (évolution de la température au cours du temps) à la fonction $q(\theta)$ (courbe d'ébullition stationnaire) s'effectue mathématiquement à l'aide de la méthode polynomiale des moindres carrés. Cette méthode porte le nom de "Problème du transfert de chaleur inversé ". Cette technique transitoire utilise en général une section d'essais de forte capacité calorifique (technique de " Hot Patch") dont l'effet est de limiter l'élévation de température et de ralentir la durée de refroidissement en freinant la propagation du front de 

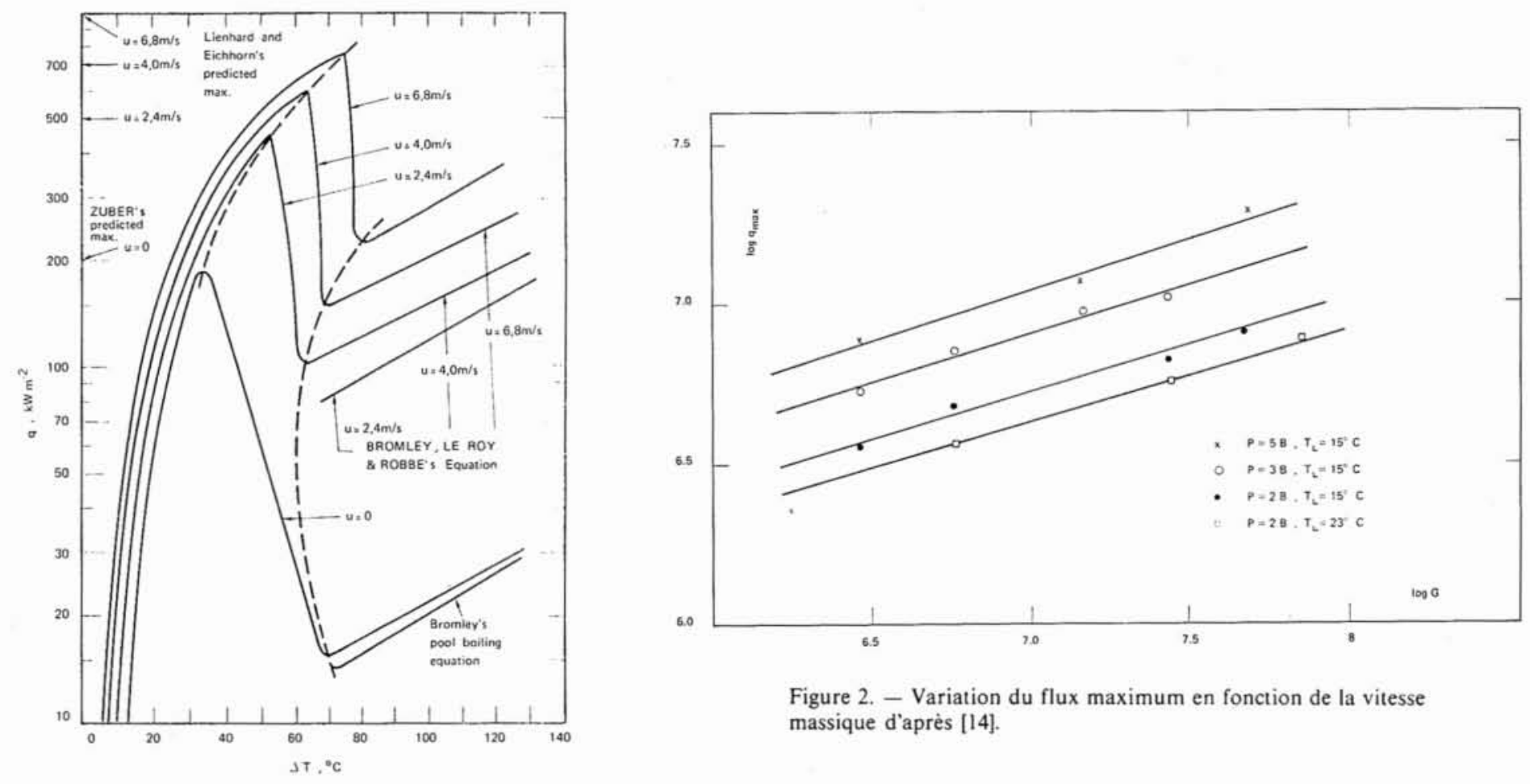

Figure 2. - Variation du flux maximum en fonction de la vitesse massique d'après [14].

Figure 1. - Influence de la vitesse massique d'après [9].

remouillage. Ainsi la courbe obtenue peut être plus aisément comparée à une courbe quasistationnaire.

Par ailleurs, l'effet de la conduction axiale au voisinage du front de remouillage peut être très important mais est difficile à évaluer.

\section{Effets paramétriques}

Nous distinguerons les paramètres dont l'effet sur les mécanismes d'ébullition est facilement quantifiable et reproductible des paramètres dont l'effet est plus aléatoire et, en tout cas, difficilement mesurable.

Dans la première classe, il s'agit des paramètres physiques définissant les conditions d'essais comme la pression, la vitesse massique, le titre ou le degré de sous-saturation, les dimensions de la section d'essais et son orientation par rapport à la gravité.

Dans la seconde classe entrent ceux caractérisant l'état de surface de la paroi, la dégradation du matériau dans le temps, l'état et l'histoire du fluide.

Compte tenu du nombre important de travaux effectués et de la dispersion des résultats, nous présentons cidessous l'influence séparée de chaque paramètre sur l'allure de la caractéristique d'ébullition.

\subsection{Influence de la vitesse massique}

L'augmentation de la vitesse massique conduit généralement à une amélioration des échanges de chaleur tout en diminuant la zone de transition. La figure I, extraite de [9] met en évidence ce phénomène dans le cas du Fréon 113. En particulier, le rapport du flux maximum au flux minimum diminue lorsque la vitesse de circulation augmente pour tendre vers 1 au-delà d'une certaine valeur de la vitesse massique. Cela implique une courbe d'ébullition monotone croissante, avec disparition complète de la zone instable. De nombreux auteurs ont ainsi vérifié la disparition du point de "burn-out" par augmentation de la vitesse massique. Stephan et Hoffmann [5], en utilisant du Fréon 114, ont observé que pour des vitesses comprises entre 1500 et $4000 \mathrm{~kg} / \mathrm{m}^{2} \mathrm{~s}$ et à des pressions variant de 5 à 15 Bar la zone de transition disparait totalement. Ces résultats sont confirmés également par les essais de Katsumata [6], Tolubinsky [7], Fukuyama [8]. La variation du flux de chaleur maximum suit une loi exponentielle avec la vitesse du type $q_{\max }$ proportionnel à $U^{\prime \prime}$ où $n=35$ dans [9], ce qui est conforme à la corrélation de Vliet et Leppert [10] ou de Kutateladze et Burakov [11] mais pas à $n=0.33$ de Lienhard et Eichhorn [12] ou Weatherhead [13]. Au cours de nos essais, nous avons obtenu une loi de puissance du flux maximum en fonction de la vitesse d'écoulement avec un exposant $\mathrm{n}$ de 0.3 [14], figure 2 . 


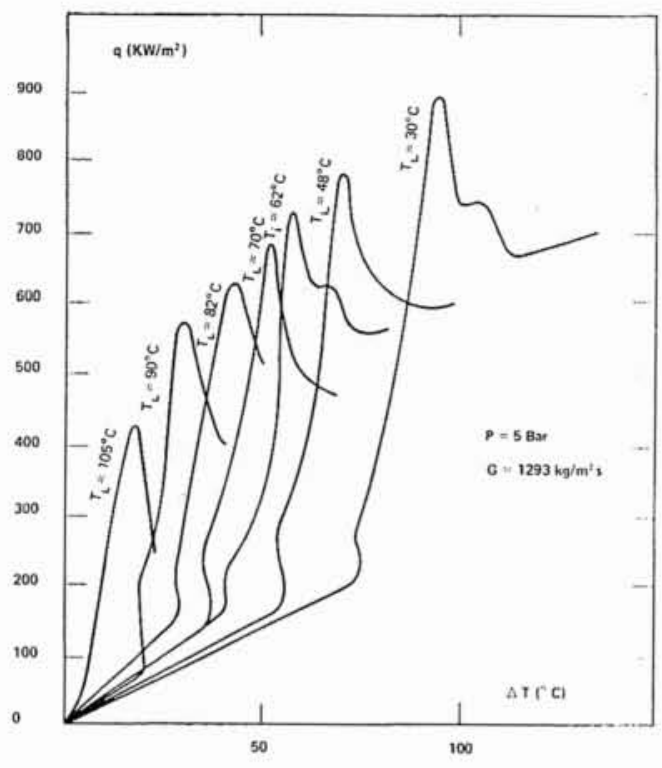

Figure 3. - Influence de la sous-saturation d'après [14].

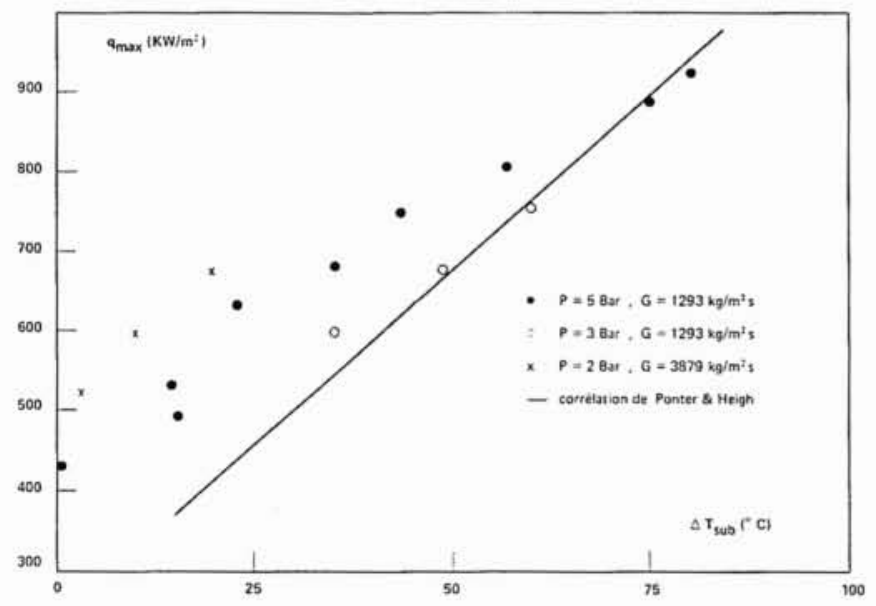

Figure 4. - Variation de $q_{\max }$ en fonction de la sous-saturation d'après [14].

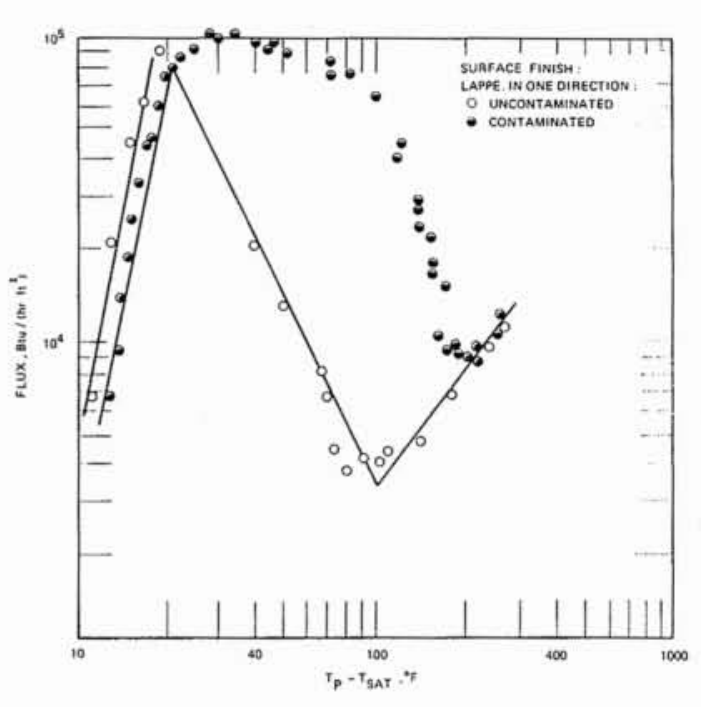

Figure 5. - Influence de l'état de la surface d'après [26].

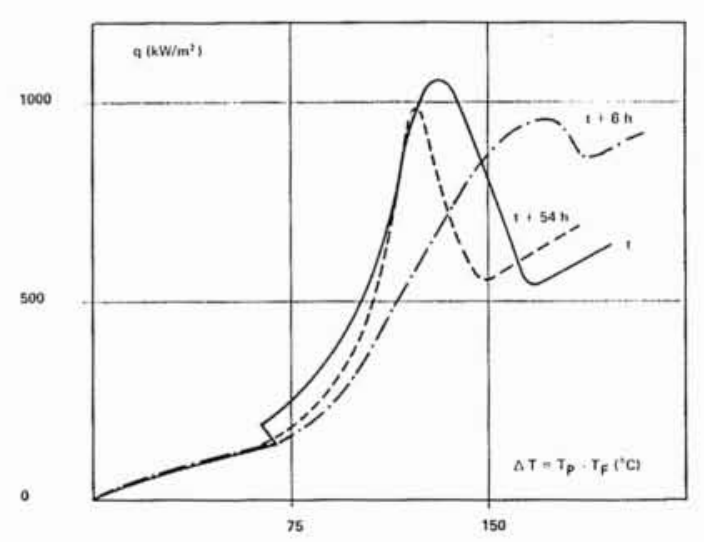

Figure 6. - Influence de la dégradation au cours du temps de l'état de la surface d'après [14]. 
Katto [15] a procédé à une analyse dimensionnelle complète permettant de corréler le flux critique en fonction de la vitesse massique et d'autres paramètres du système (voir 3.1.3).

\subsection{Influence de la pression}

L'augmentation de pression convective et d'ébullition nucléée [16];

- une amélioration du transfert dans la zone de transition $[5,16]$.

Hesse [2] a montré qu'une élévation de pression augmentait la pente $\left|\frac{\partial q}{\partial \theta}\right|$ de la zone de transition, ce qui rend le processus de stabilisation plus difficile et le transfert thermique moins efficace. Plummer [17] a observé l'augmentation de $\Delta T_{\min }$ avec la pression dans le cas d'un tube vertical fonctionnant en régime transitoire. Des lois de puissance $q_{\max } \sim p^{m}$ ont été proposées par Labuntzov [18] avec $m=0.15$ et par Kutateladze [19] avec $m=0.5$.

\subsection{Influence de la sous-saturation et du titre}

La sous-saturation a peu d'influence dans la zone d'ébullition nucléée car l'écart entre deux courbes correspond à l'échauffement supplémentaire de la paroi nécessaire pour atteindre la température de saturation. Cet effet peut être interprété en évaluant la quantité de chaleur $q$ emportée par une bulle de rayon $R_{\max }$ et de fréquence $f$ :

$$
q \sim \rho_{L} \cdot C_{L} \cdot f \cdot R_{\max }^{3}\left[\Delta T_{\text {sat }}+\Delta T_{\text {sub }}\right]
$$

Il a été montré [24] que $q$ ne variera pas beaucoup avec la sous-saturation puisque d'après [16] $R_{\max }$ et $f$ diminuent quant $\Delta T_{\text {sub }}$ augmente. L'augmentation de la sous-saturation contribue à une amélioration de l'échange dans la zone de transition [17, 20,21] et à une augmentation du rapport $q_{\max } / q_{\min }$. Ceci conduit à la disparition de la zone de transition pour des titres positifs élevés (courbes monotones croissantes) [7, 8, 22]. Par ailleurs $q_{\max }$ augmente avec la sous-saturation ou le nombre de Jakob

$$
J_{a}=\frac{\rho_{L} \cdot C_{L} \cdot \Delta T_{\text {sub }}}{\rho_{v} \cdot h_{f s}},
$$

comme il a été mis en évidence dans [26].

La figure 3 extraite de la référence [14] montre le premier des effets mentionnés ci-dessus, tandis que la figure 4, montre la variation de $q_{\max }$ avec la sous-saturation. Le caractère linéaire des résultats présentés est à comparer avec la corrélation de Ponter et Haigh [23].

\subsection{Influence de l'état de surface}

De nombreux facteurs liés à l'état de surface influencent les mécanismes de transfert de chaleur. Un nombre important de travaux empiriques existent sur ce sujet, mais compte tenu de la difficulté à définir un paramètre caractéristique de l'état de la surface, les conclusions ne sont pas rigoureuses et ne peuvent indiquer que des tendances. Nous ne ferons pas un examen exhaustif de l'influence de ces paramètres mais nous donnerons simplement quelques exemples significatifs extraits de la littérature.

\subsubsection{Rugosité de la surface}

La majorité des travaux montre que la rugosité a peu d'influence sur la valeur du flux maximum et du flux minimum [26]. De plus, ce paramètre influence la valeur de la température $\Delta T_{\max }$ correspondant au pic de la courbe sans faire varier celle de $\Delta T_{\min }$. Plus la rugosité est importante, plus la différence $\left(\Delta T_{\min }-\Delta T_{\max }\right)$ devient grande. (Hesse [2], Brown [27] qui, de plus, a mis en évidence l'influence de la position des stries par rapport à l'écoulement).

$\mathrm{Ce}$ paramètre est donc important pour la zone de transition et en conséquence sur la stabilité du processus.

\subsubsection{Processus physiques de la surface}

Certains auteurs, comme Plummer [17], ont pu montrer que les propriétés de transfert dépendent du rapport $(\rho \cdot C \cdot \lambda)_{L} /(\rho \cdot C \cdot \lambda)_{p}$. En particulier, le coefficient d'échange dans la zone de transition est d'autant plus élevé que $(\rho \cdot C \cdot \lambda)_{p}$ est plus faible. L'expression de $h$, le coefficient d'échange, est directement reliée à $(\rho \cdot C \cdot \lambda)$, [28].

\subsubsection{Revêtement de la surface}

L'influence d'un revêtement a pour effet d'augmenter la surchauffe $\Delta T_{\text {sat }}$ à flux constant [29] ainsi que la valeur du flux minimum. On peut ainsi envisager la disparition complète de la zone de transition. Certains auteurs [28, 30] ont trouvé l'apparition d'un second maximum dans la zone de transition, laissant apparaître une courbe d'ébullition en forme de "selle".

\subsubsection{Contamination, oxydation, dégradation}

Berenson [26] a montré que la contamination de la surface était la cause de la différence entre les deux courbes de la figure 5. D'une manière générale, l'allure de la courbe d'ébullition est profondément affectée par une oxydation ou un agent polluant de la surface. L'influence de la dégradation de l'état de surface au cours du temps sur les mécanismes d'échange de chaleur, en particulier pour des expériences conduites en Fréon 113, est parfaitement illustrée sur la figure 6 [14]. On trouve des résultats analogues dans les références [2,31]. Plummer [17] et Iloeje [32] ont trouvé que l'oxydation de la surface peut influencer l'ébullition de transition parce que :

- la conductivité thermique du matériau diminue,

- la rugosité de la paroi augmente,

- l'angle de contact liquide-paroi diminue,

ces trois modifications conduisant à une augmentation de la valeur de $\left(\Delta T_{\min }\right)$.

\subsection{Influence d'autres paramètres}

Beaucoup d'autres paramètres ont une influence non négligeable sur les mécanismes de transfert et, par conséquent, sur la forme de la courbe d'ébullition. On peut citer, par exemple, la géométrie et les dimensions du système 
chauffé, son orientation par rapport à la gravité, la présence de gaz non condensables dans le fluide en ébullition, etc. Nous ne citerons qu'un seul paramètre affectant l'allure de la courbe et les travaux s'y rapportant : il s'agit de la méthode de chauffage. Ainsi, dans [33, 34], on note que les auteurs ont trouvé des valeurs de flux maximums différentes selon que le chauffage est électrique (effet Joule) ou à température de paroi imposée par un fluide auxiliaire.

\section{Corrélations et construction de courbes}

\subsection{Corrélations utilisables}

Avant de présenter quelques courbes d'ébullition construites numériquement, nous donnons dans ce paragraphe les formules de corrélations recensées dans la bibliographie pour les différents régimes de transfert apparaissant à flux de chaleur croissant.

\subsubsection{Convection forcée en phase liquide}

En régime turbulent, la corrélation couramment utilisée est celle de Dittus - Boelter [35] :

$$
N u=0.023\left(R e_{L}\right)^{0.8} \cdot\left(P r_{L}\right)^{0.33}
$$

\subsubsection{Ebullition nucléée}

Dans ce régime, on utilise la formule de Chen [36]

$$
\begin{aligned}
h= & 0.00122 \frac{\lambda_{L}^{0.79} \cdot C_{L}^{0.45} \cdot \rho_{L}^{0.49}}{\sigma^{0.5} \cdot \mu_{L}^{0.29} \cdot h_{f 8}^{0.24} \cdot \rho_{r}^{0.24}} \\
& \left(T_{p}-T_{\mathrm{sat}}\right)^{0.24} \cdot(\Delta p)^{0.75} \cdot S \\
+ & 0.023\left[\frac{D . G(1-x)}{\mu_{L}}\right]^{0.8} \cdot\left[\frac{C_{L} \cdot \mu_{L}}{\lambda_{L}}\right]^{0.4} \cdot \frac{\lambda_{L}}{D} \cdot F
\end{aligned}
$$

valable pour : $0.09<P<6.9 \mathrm{MPa}$;

$54<G<4070 \frac{\mathrm{kg}}{\mathrm{m}^{2} \cdot \mathrm{S}} ; 0<x<0.7$

En sous-saturation, la formule de Chen se trouve modifiée de la façon suivante :

$q=0.00122 \frac{\lambda_{L}^{0.79} \cdot C_{L}^{0.45} \cdot \rho_{L}^{0.49}}{\sigma^{0.5} \cdot \mu_{L}^{0.29} \cdot h_{f /}^{0.24} \cdot \rho_{,}^{0.24}}$

$$
\left(T_{p}-T_{\mathrm{sat}}\right)^{1.24} \cdot(\Delta p)^{0.75} \cdot S
$$

$+0.023\left(\frac{\lambda_{L}^{0.6} \cdot G^{0.8} \cdot C_{L}^{0.4}}{\mu_{L}^{0.4} \cdot D^{0.2}}\right) \cdot\left(T_{\rho}-T_{L}\right)$

Ces formules sont d'ailleurs aussi bien valables en convection forcée qu'en convection naturelle. Les paramètres $F$ et $S$ qui apparaissent dans la corrélation sont donnés par les expressions suivantes :

$$
\begin{aligned}
& 1.0 \quad x_{n}^{-1} \leqslant 0.1 \\
& F= \\
& 2.35\left(x_{u}^{-1}+0.213\right)^{0.735} \quad x_{u}^{-1}>0.1 \\
& {\left[1+0.12\left(\operatorname{Re}^{\prime}\right)^{0.14}\right]^{-1} \quad R e^{\prime}<32.5} \\
& S=\quad\left[1+0.42\left(R e^{\prime}\right)^{0.78}\right]^{-1} \quad 32,5<R e^{\prime}<50 \\
& 0.1 \quad R e^{*}>50
\end{aligned}
$$

où

$$
x_{n}^{-1}=\left(\frac{x}{1-x}\right)^{0.9} \cdot\left(\frac{\rho_{L}}{\rho_{r}}\right)^{0.5} \cdot\left(\frac{\mu_{i}}{\mu_{L}}\right)^{0.1}
$$

est l'inverse du paramètre de Lockhart

- Martinelli, et

$$
R e^{\prime}=\frac{G(1-x) D}{\mu_{L}} \cdot F^{1.25} \cdot 10^{-4}
$$

pour l'ébullition saturée et

$\frac{G \cdot D}{\mu_{L}} \cdot 10^{-4}$ pour l'ébullition sous-saturée, oủ le facteur multiplicatif $10^{-4}$ n'apparait que pour faciliter les calculs.

\subsubsection{Flux critique}

Dans le cas de l'ébullition stagnante, on emploie l'équation de Zuber [37]

$$
q_{\max }=\frac{\pi}{24} \sqrt{\rho_{r}} \cdot h_{f x} \cdot \sqrt[4]{g \cdot \sigma \cdot\left(\rho_{L}-\rho_{r}\right)}
$$

et, pour l'ébullition en convection forcée, les corrélations de Katto [15] :

- ébullition saturée :

$q_{m s}$

$=0.1 \mathrm{G} \cdot h_{f s} \cdot\left(\frac{\rho_{r}}{\rho_{L}}\right)^{0.133} \cdot\left(\frac{\sigma \cdot \rho_{L}}{G^{2} \cdot L}\right)^{0.33} \cdot \frac{1}{1+0.0031 L / D}$

- ébullition sous-saturée :

$$
\begin{aligned}
& q_{\max }=q_{m s}\left(1+K \cdot \frac{\Delta h}{h_{f s}}\right) \\
& \text { où } \quad K=\frac{5}{6} \cdot \frac{0.0124+D / L}{\left(\frac{\rho_{r}}{\rho_{L}}\right)^{0.133} \cdot\left(\frac{\sigma \cdot \rho_{L}}{G^{2} \cdot L}\right)^{0.33}} \\
& \text { titre } x=\frac{4 q_{m s} \cdot L}{G \cdot h_{f / k} \cdot D}-\frac{\Delta h}{h_{f k}}
\end{aligned}
$$

\subsubsection{Ebullition en film}

L'équation de Bromley [38] est valable dans le cas de l'ébullition stagnante; elle prend la forme suivante :

$$
h_{f}=0.62\left[\frac{\lambda_{r}^{3} \cdot \rho_{r} \cdot g \cdot\left(\rho_{L}-\rho_{v}\right) h_{f s}^{\prime}}{\mu_{v} \cdot \Delta T \cdot L^{\prime}}\right]^{0.25}
$$

où $L^{\prime}$ est la première longueur d'onde critique de l'instabilité de Taylor.

$$
L^{\prime}=2 \pi\left[\sigma \cdot g^{-1} \cdot\left(\rho_{L}-\rho_{1}\right)^{-1}\right]^{0.5}
$$


L'expression de la chaleur latente figurant dans la formule de Bromley correspond à la formule de Kutateladze :

$$
h_{f \mathrm{R}}^{\prime}=h_{f s}+0.5 C_{p r}\left(T_{p}-T_{s a t}\right)
$$

En convection forcée, on utilisera l'équation de Dougall-Rohsenow :

$$
h_{f}=0.023 \frac{\lambda_{r}}{D}\left[\operatorname{Re}_{s}\left\{x+\left(1-x \frac{\rho_{r}}{\rho_{L}}\right\}\right]^{0.8} \cdot\left(\operatorname{Pr}_{r}\right)^{0.4}\right.
$$

\subsubsection{Température de Leidenfrost}

Citons la formule de Séméria [39]

$$
T_{\min }=\frac{27}{32} T_{c r}+\sqrt{\frac{(\rho \cdot C \cdot \lambda)_{L}}{(\rho \cdot C \cdot \lambda)_{p}}}\left(\frac{27}{32} T_{c r}-T_{L}\right)
$$

ou l'équation de Berlin et al [40]

$$
\begin{aligned}
\frac{T_{\min }-T_{\text {sat }}}{T_{c r}-T_{\text {sat }}}=0.165 & +2.5\left[\frac{(\rho \cdot C \cdot \lambda)_{L}}{(\rho \cdot C \cdot \lambda)_{p}}\right]^{0.25} \\
& +\frac{(\rho \cdot C \cdot \lambda)_{L}}{(\rho \cdot C \cdot \lambda)_{p}}
\end{aligned}
$$

qui sont fonction des propriétés physiques du liquide et de la paroi.

\subsection{Programme numérique}

L'algorithme du programme, figure 7 , analogue à celle de Bjornard et Griffith [41] contient toutes les corrélations citées précédemment valables pour le Fréon 113 sans application du principe de similitude [42]. Les données nécessaires au démarrage du programme correspondent aux conditions expérimentales d'essai : ce sont la pression, le débit massique, le titre en vapeur, la température d'entrée du fluide, la géométrie de la section d'essai (longueur et diamètre), les propriétés physiques de la phase liquide et de la phase vapeur, ainsi que celles de la paroi. Il faut noter que celui-ci ne permet pas de tracer la zone de transition faute de corrélations satisfaisantes. Celle-ci est assimilée à une droite une fois déterminés numériquement le point de flux maximum et celui de Leidenfrost. Les calculs sont arrêtés lorsque l'écart de température paroi-liquide atteint une certaine valeur, choisie dans le cas présent égale à $150^{\circ} \mathrm{C}$.

\subsection{Analyse de quelques résultats}

\subsubsection{Courbes d'ébullition}

Quelques courbes construites à l'aide de l'algorithme ci-dessus sont présentées sur les figures 8 à 11 et comparées avec celles obtenues par certains auteurs dans les mêmes conditions expérimentales d'essais. On notera une similarité acceptable entre les courbes théoriques et expérimentales.

Néanmoins, ces corrélations ne sont pas d'un emploi aisé et ne s'appliquent pas à toutes les configurations et à tout type de liquide. Une recherche dans cette direction

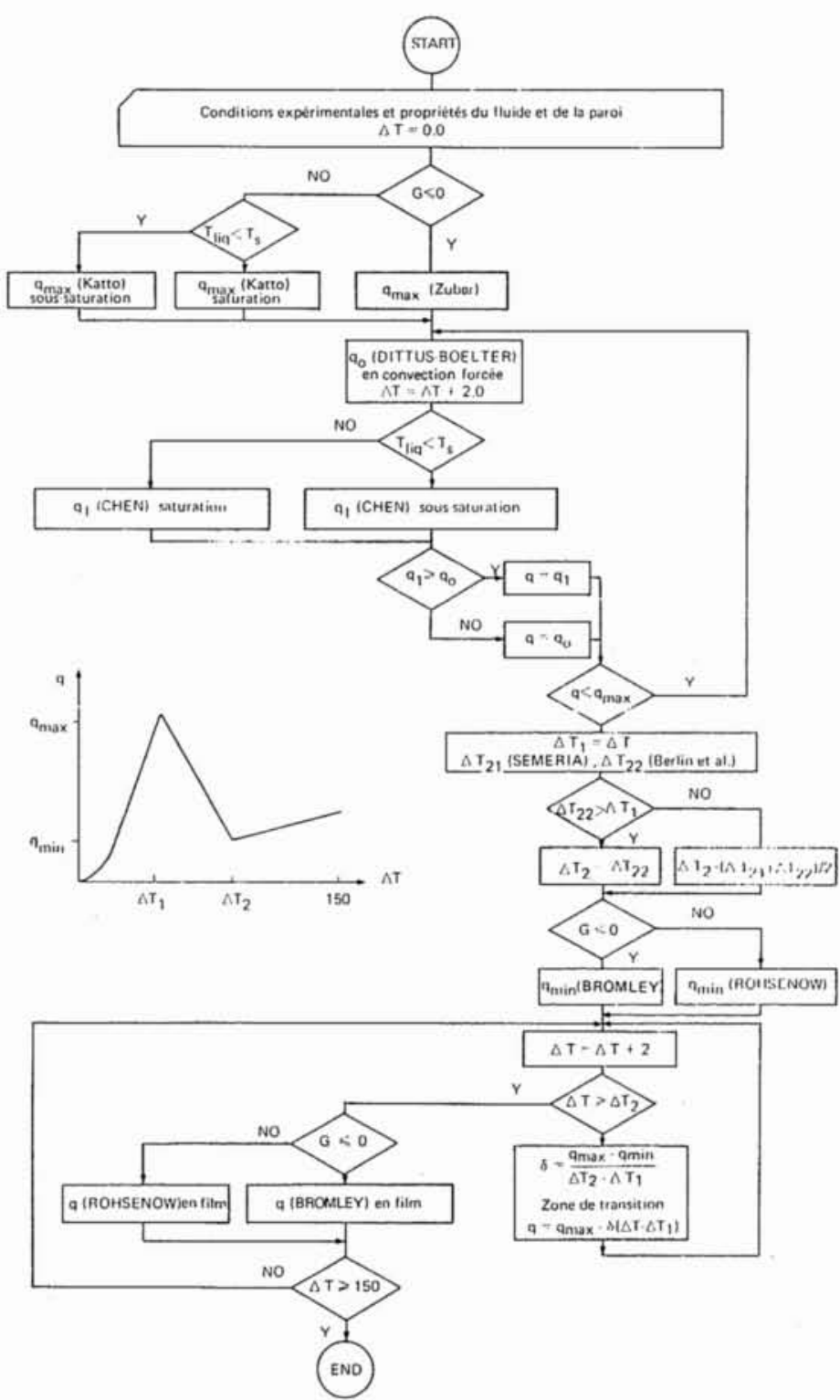

Figure 7. - Organigramme du programme de tracé des courbes d'ébullition.

demeure nécessaire aujoud'hui afin, en particulier, d'obtenir des grandeurs adimensionnelles dont l'usage serait évidemment plus fiable dans certains codes de calcul [1]. 
470

LA HOUILLE BLANCHE/N ${ }^{\circ}$ 6-1986

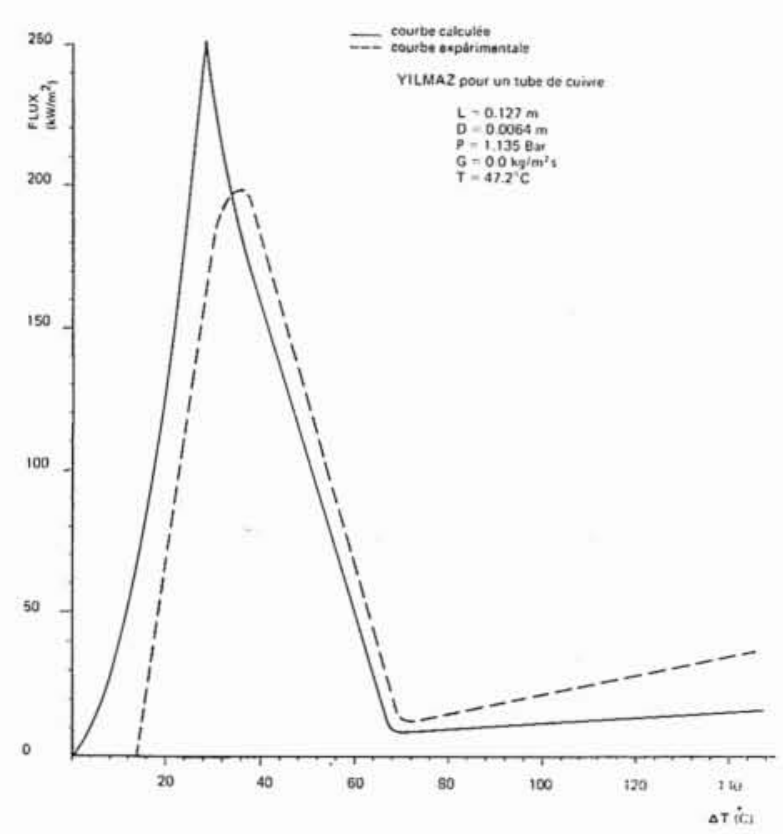

Figure 8. - Comparaison entre une courbe d'ébullition calculée et une expérimentale d'après [9].

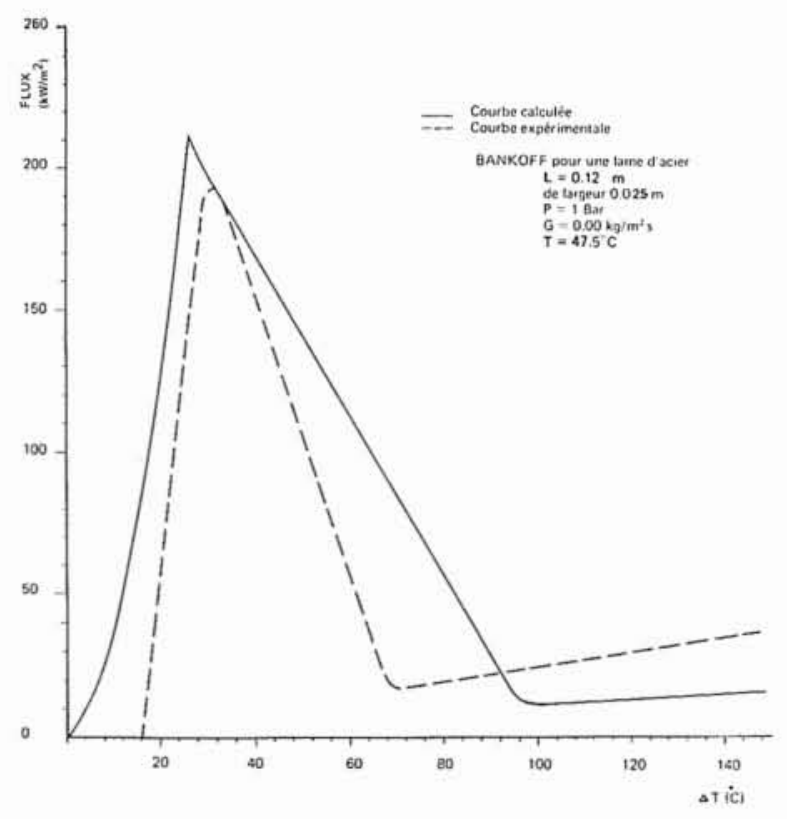

Figure 10. - Comparaison entre une courbe d'ébullition calculée et une expérimentale d'après [51].

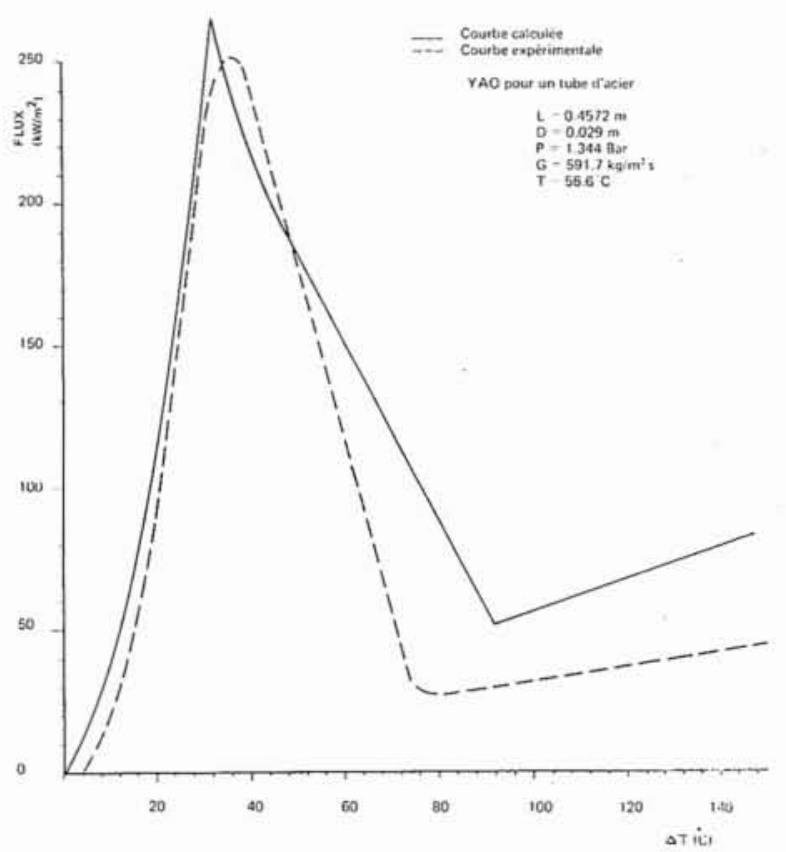

Figure 9. - Comparaison entre une courbe d'ébullition calculée et une expérimentale d'après [50].

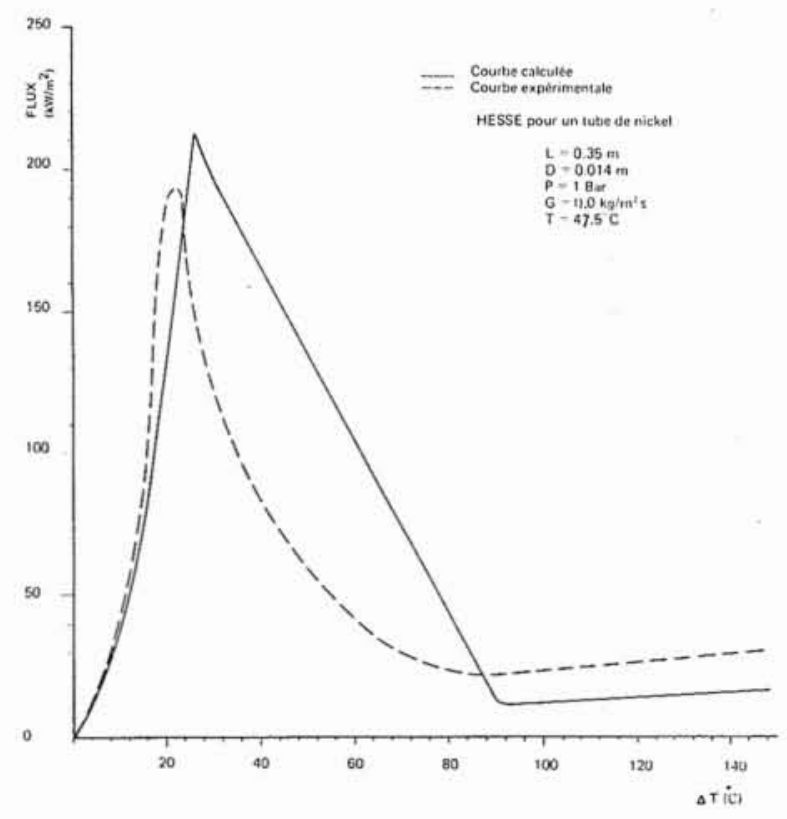

Figure 11. - Comparaison entre une courbe d'ébullition calculée et une expérimentale d'après [2]. 


\subsubsection{Effets paramétriques}

Les figures 12 à 15 illustrent l'effet de quatre paramètres :

Figure 12: Effet de la vitesse massique $G$

La variation de $G$ n'influe pas beaucoup sur la zone d'ébullition nucléée, comme l'ont trouvé Stevens [43], Hildebrandt [45] et Kutaladze [46]. Le flux critique augmente, figure 12 , en accord avec les résultats présentés dans $[5,9,44]$ ou diminue, figure 12 bis, avec $G$ augmentant suivant que l'on opère en sous-saturation ou aux titres positifs respectivement.

La zone de transition se trouve donc translatée vers le haut ou le bas suivant qu'il s'agit de la première ou la deuxième situation. Dans les deux cas, le rapport $q_{\max } / q_{\min }$ diminue à vitesse massique croissante, ce qui conduit à la disparition totale de la zone de transition pour une certaine valeur critique de $G$, différente pour chaque cas. Enfin, l'augmentation de $G$ conduit à une amélioration du transfert dans la zone en film (Greif [24], Stevens [43] et Chen [47].

Figure 13: effet de la pression.

On peut noter que l'accroissement de la pression améliore le coefficient nucléée et augmente le flux critique, comme l'ont remarqué Stephan [5] et Hildebrandt [45]. Les avis divergent sur les raisons de l'augmentation du flux en zone de transition (Hesse [2], Stephan [5], Ellion [16]). Il n'existe, à notre connaissance, aucun résultat sur l'effet de pression en ébullition en film, excepté au voisinage de la pression critique.

Figure 14 : effet de la sous-saturation et du titre

L'augmentation de la sous-saturation a peu d'influence sur la zone d'ébullition nucléée (fig. 14) comme nous l'avons évoqué précédemment. Elle semble, par contre, conduire à une amélioration de l'échange dans la zone de transition, ainsi que l'on vérifié Ellion [16], Ragheb [20] et Cheng [21]. Le flux maximum augmente linéairement avec le degré de sous-saturation (Gentile [48], Katto [49]). D'autre part, on vérifie la disparition complète de la zone de transition à titre croissant (fig. 14bis) comme mentionné dans 2.3.

\section{Figure 15 : effet de la géométrie}

L'augmentation du rapport $L / D$ de la section d'essai conduit à une diminution du flux critique, en accord avec les résultats de Stevens [43] et Katto [49], et à une amélioration de transfert de chaleur en ébullition nucléée et en film.

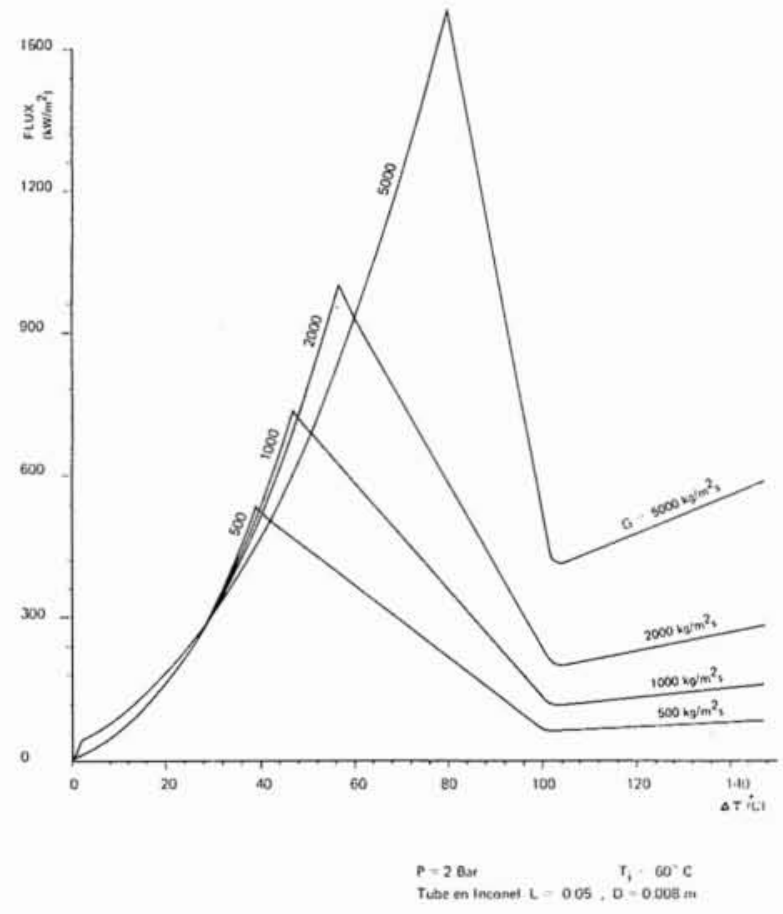

Figure 12. - Influence de la vitesse massique en sous-saturation.

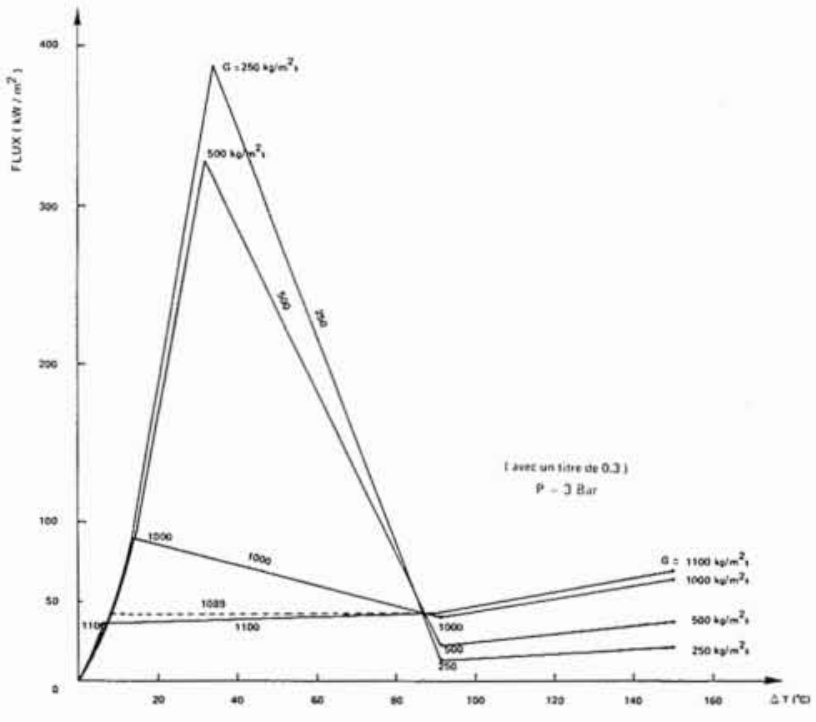

Figure 12 bis. - Influence de la vitesse massique (avec un titre de 0.3 ). 


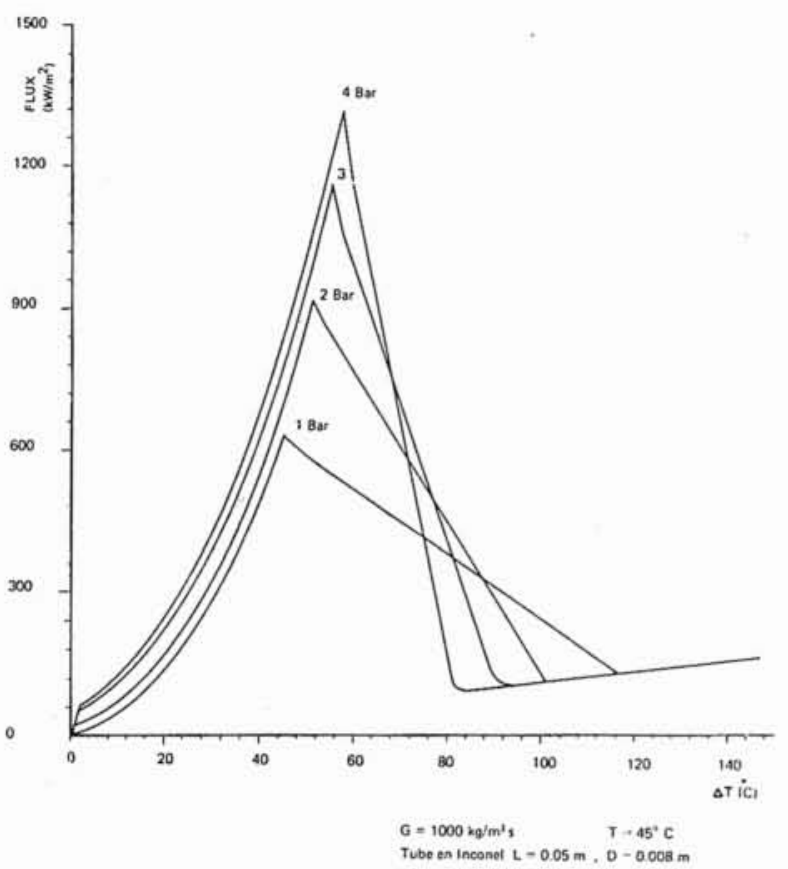

Figure 13. - Influence de la pression.

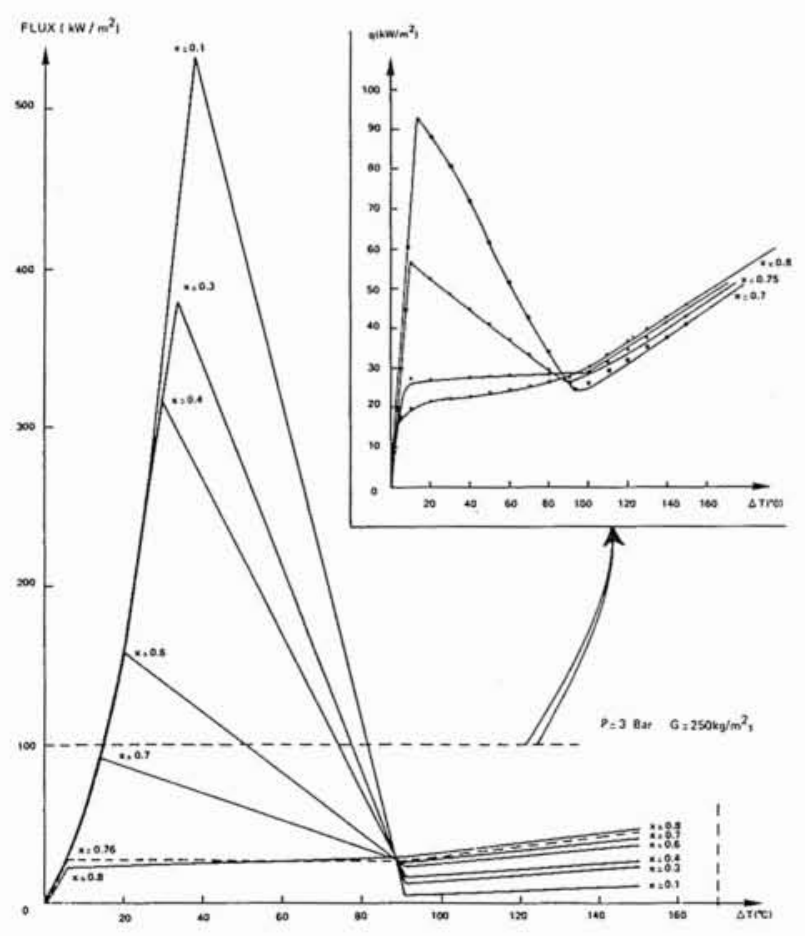

Figure 14 bis. - Influence du titre.

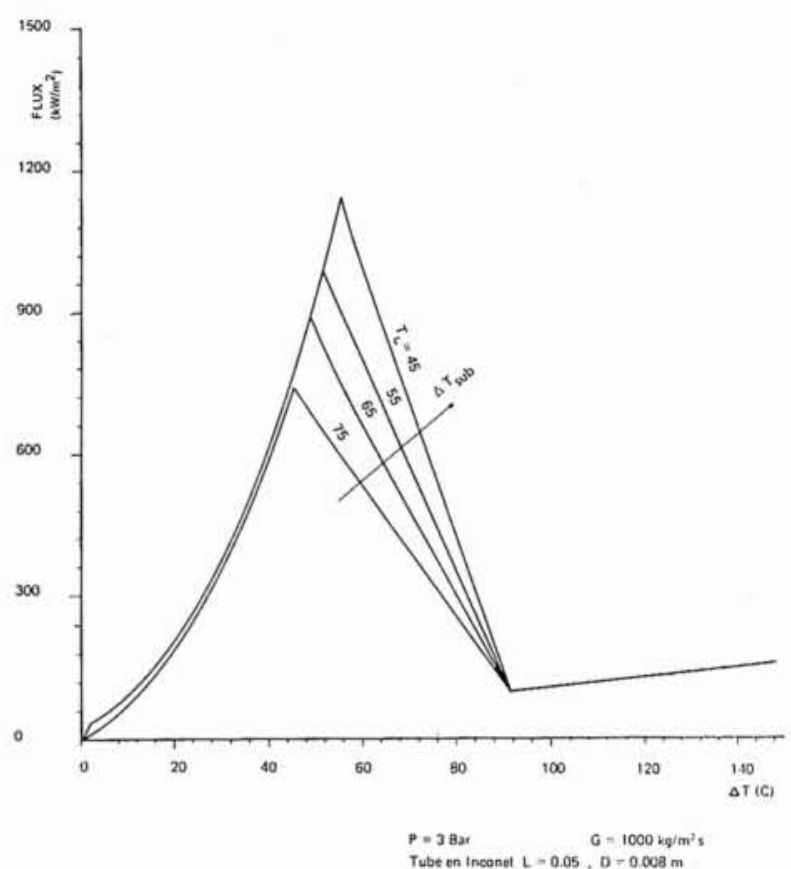

Figure 14. - Influence de la sous-saturation.

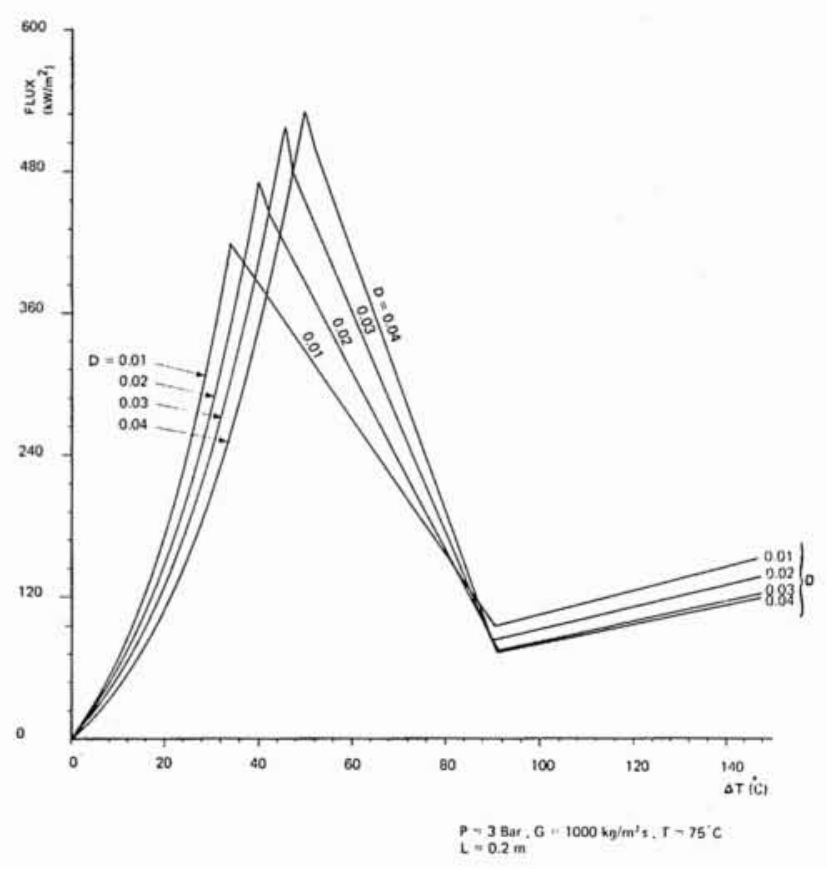

Figure 15. - Influence du rapport L/D. 


\section{Conclusion}

Dans cet article et le précédent [1], nous avons effectué une revue générale des principaux résultats disponibles concernant les mécanismes de l'ébullition en convection forcée, représentés globalement par une courbe d'ébullition.

Celle-ci, dont la notion est liée à celle de stabilité thermique, n'est pas aisée à obtenir dans sa totalité, compte tenu de l'instabilité liée à la crise d'ébullition et de l'influence importante de nombreux paramètres, dont certains sont difficilement contrôlables.
Ainsi, en dépit d'un nombre considérable de travaux effectués sur le sujet, il demeure nécessaire de continuer à développer des recherches précises, tant expérimentales que théoriques, afin de pouvoir aboutir à une modélisation correcte de ces mécanismes.

C'est en ce sens que nous travaillons à l'ENSTA et à l'EDF, expérimentalement en perfectionnant l'usage d'un asservissement électronique, théoriquement en tentant de présenter l'influence des différents paramètres sous forme de courbes adimensionnelles.

\section{NOMENCLATURE}

\section{Capacité calorifique}

$D$ Diamètre du tube

$f$ Fréquence de bulle

$F$ Facteur correctif de Chen

$g$ Accélération de la pesanteur

$G$ Vitesse massique

$h$ Coefficient de transfert thermique

$\Delta h$ Différence d'enthalpie

$h_{f s}$ Chaleur latente de vaporisation

$K$ Constante de Katto

$n$ Exposant

$\mathrm{Nu}$ Nombre de Nusselt

$L \quad$ Longueur du tube

$L$. Longueur d'onde critique de l'instabilité de Taylor

Ja Nombre de Jakob

p Pression

Pr Nombre de Prandtl

$q$ Densité de flux thermique

$R$ Rayon de bulle

$R e$ Nombre de Reynolds

$S$ Facteur correctif de Chen

$t$ Temps

$T$ Température

\begin{tabular}{|c|c|c|}
\hline $\mathrm{J} / \mathrm{kg}^{\circ} \mathrm{C}$ & $U$ & Vitesse \\
\hline $\mathrm{m}$ & $x$ & Titre en vapeur \\
\hline$s^{-1}$ & $\Delta p$ & Différence de pression \\
\hline $\mathrm{m} / \mathrm{s}^{2}$ & $\begin{array}{l}\Delta T \\
\theta\end{array}$ & $\begin{array}{l}\text { Différence de température } \\
\text { Ecart de température paroi-fluide }\end{array}$ \\
\hline $\mathrm{kg} / \mathrm{m}^{2} \mathrm{~s}$ & $\lambda$ & Conductibilité thermique \\
\hline $\mathrm{W} / \mathrm{m}^{2}{ }^{\circ} \mathrm{C}$ & $\mu$ & Viscosité dynamique \\
\hline $\mathrm{J} / \mathrm{kg}$ & $\rho$ & Masse volumique \\
\hline \multirow[t]{2}{*}{$\mathrm{J} / \mathrm{kg}$} & $\sigma$ & Tension superficielle \\
\hline & Indic & \\
\hline $\mathrm{m}$ & cr & critique \\
\hline & e & externe \\
\hline & $\begin{array}{l}j \\
i\end{array}$ & $\begin{array}{l}\text { film } \\
\text { interne }\end{array}$ \\
\hline $\mathrm{N} / \mathrm{m}^{2}$ & $L$ & liquide \\
\hline & $\min$ & point de Leidenfrost \\
\hline $\mathrm{W} / \mathrm{m}^{2}$ & $\max$ & maximum \\
\hline & $p$ & paroi \\
\hline & sat & saturation \\
\hline & sub & sous-saturation \\
\hline & $v$ & vapeur \\
\hline
\end{tabular}

$\mathrm{m} / \mathrm{s}$

$\mathrm{N} / \mathrm{m}^{2}$

${ }^{\circ} \mathrm{C}$

${ }^{\circ} \mathrm{C}$

$\mathrm{W} / \mathrm{m}^{\circ} \mathrm{C}$

$\mathrm{kg} / \mathrm{ms}$

$\mathrm{kg} / \mathrm{m}^{3}$

$\mathrm{N} / \mathrm{m}$

\section{Références}

[1] LLORRY M. GENTILE D., AFIFY M. - Courbes d'ébullition en convection forcée. Première partie : revue bibliographique, La Houille Blanche, $\mathrm{n}^{\circ}$ 6-7, (1985).

[2] HESSE G. - Heat transfer in nucleate boiling, maximum heat flux and transition boiling, Int. J. of Heat \& Mass Transfer, 16, p. 1611, (1973).

[3] BENEJEAN R., LLORY M. - Stabilisation électronique d'un processus thermique non linéaire, $E D F$, Bulletin de la $D E R$, Série $A, \mathrm{n}^{\circ} 3$, p. 43 (1981).

[4] GENTILE D., LLORY M., BENEJEAN R. - Stabilisation of Freon 113 Subcooled Boiling in Forced Convection, Int. Com.
Heat \& Mass Transfer, 12, n 5, p. 521, (1985)

[5] STEPHAN K., HOFFMAN EG. - Transition and Flow Boiling Heat Transfer inside a horizontal tube. Int. J. of Heat \& Mass Transfer, 20, p. 1381, (1977).

[6] KATSUMATA 1., HIRATA K. - Lébullition nucléée et la disparition du phénomène d'assèchement en présence d'un écoulement d'eau ultra-rapide dans un tube, traduit de Trans. of the Japan Soc, JSME, Série 2, Vol.43, № 375, p. 4257, (1977)

[7] TOLUBINSKY V.I. et al. - Temperature of the pipe wall in transition from nucleate to film boiling, Heat Transfer Soviet Research, Vol 15, №1 (1983) 
[8] FUKUYAMA Y. HIRATA M. - Boiling Heat Transfer Characteristics with high mass flux and disappearance of $\mathrm{CHF}$ following to DNB, 7th Int. Heat Trans. Conf., Münich, Vol 4, p. 273, (1982)

[9] YILMAZ S., WESTWATER J.W. - Effect of velocity on Heat Transfer to Boiling Freon 113, J. Heat Transfer 102, p. 26 , (1980)

[10] VLIET G., LEPPERT G. - Critical Heat Flux in Nearly Saturated Water flowing normal to a cylinder, Trans. of ASME, 86, p. 68, (1964)

[11] KUTATELADZE SS., BURAKOV BA. - The critical Heat Flux for Natural Convention and Forced Flow of Boiling and Subcooled Dowtherm, Problems of Heat Transfer and $\mathrm{Hy}$. draulics of Two-phase Media, Pergamon Press, Oxford, p. 63 , (1969)

[12] LIENHARD JH., EICHHORN R. - Peak Boiling Heat Flux on Cylinders in a Cross-Flow, Int. J. of Heat \& Mass Transfer, 19, p. 1135, (1976)

[13] WEATHERHEAD RT. - Boiling Burnout Heat Flux for Wires in Water Crossflow at atmospheric pressure, Boiling Burnout Progress Report n ${ }^{\circ} 5$, Argonne national Laboratory, (1955)

[14] GENTILE D. - Etude expérimentale de l'ébullition du Fréon 113 en convection forcée à l'aide d'un dispositif électronique de stabilisation. Rapport ENSTA/GPI de fin de Contrat DER/EDF, (1984)

[15] KATTO Y. - A generalized correlation of critical heat flux for the forced convection boiling in vertical uniformly heated round tubes, Int. J. of Heat \& Mass Transfer, 21, 1527, (1978)

[16] ELLION ME. - A study of the mechanism of boiling heat transfer, Jet Propulsion Laboratory, California Institute of Technology USA, (1954)

[17] PLUMMER D. et al. - A study of post critical heat flux hea transfer in a forced convection system, Rapport DSR 73645-80, Department of Mechanical Engineering, Cambridge (1973)

[18] LABUNTZOV D.A. et al. - Critical heat fluxes in boiling at low pressure region, Proc. of the 3rd Int. Heat Transf. Conf., p. 221, (1966)

[19] KUTATELADZE SS. Heat Transfer Theory Foundations, Nauka, (1970)

[20] RAGHEB HS., CHENG SC., GROENEVELD DC. Observation in Transition Boiling of subcooled water under forced convective conditions, Int. J. of Heat \& Mass Transfer 24 , p. $1127,(1981)$

[21] CHENG SC., CHENG K. - Measurements of Boiling curves of subcooled water under forced convective conditions, Int. J. of Heat \& Mass Transfer, 21, p. 1385, (1978)

[22] KEARSEY H.A. - Steam water Heat Transfert post-burnout conditions, Chem. Proc. Eng. p. 455, (1985)

[23] PONTER AB., HAIGH CP. - Boiling Crisis in saturated and subcooled pool boiling at reduced pressures, Int. J. of Heat \& Mass Transfer, 12, p. 429, (1969).

[24] GREIF R. et al. - Heat Transfer to a Boiling Liquid Mechanisms and Correlations, J. of Heat Transfer, 43, (1959)

[25] WITTE LC., LIENHARD JH. - On the existence of two transition boiling curves, J. Heat \& Mass Transfer, 25, 771, (1982)

[26] BERENSON PG. - Transition Boiling Heat Transfer from a horizontal surface, MIT Report, Cambridge, (1960)

[27] BROWN W. - Study of flow surface boiling, Ph. d. Massachussets Inst. of Technology, Cambridge, (1967)

[28] GRIGOR VA. et al. - Film and Transition Boiling Heat Transfer to Cryogenic Fluids, Heat Transfer, Soviet Research, $11, \mathrm{n}^{\circ} 6,(1979)$
[29] MADDOCK, JAMES, NORRIS. - Superconductive Composites: Heat Transfer and Steady State Stabilisation, Cryogenics, p. 261, (1969)

[30] NISHIKAWA K., HASEGAWA S., HONDA H. - Studies on Boiling Characteristic Curve, Mem. Fac. Engng. Kyushu. Univ., 27, 133, (1967)

[31] AKAGAWA K. et al. - Influence of fouling on boiling heat transfer to cryogenic coolant, Heat Transf. Conf., Tokyo, Vol 4 , p. $25,(1974)$

[32] ILOEJE OC. et al. - An investigation of the collapse and surface rewet in film boiling in forced vertical flow, Trans. ASME, p. 166, (1975)

[33] FRANCE DM. - DNB in liquid metal heated forced convection boiling, Int. J. of Heat \& Mass Transfer, 16 p. 2343 , (1973)

[34] CUMO M. et al. - Temperature controlled and Heat Flux controlled systems, La Termotecnica, Vol XXXI, n 6, p. 313 (1977)

[35] COLLIER JG. - Two-phase Flow and Heat Transfer in the power and process industries, H.P.C. p. 233, (1981)

[36] CHEN JC. - Correlation for boiling Heat Transfer to saturated liquids in convective Flow, Int. Eng. Chem. Proces Design and Development, 5, p. 322, (1966)

[37] ZUBER N. - Discussion of paper by Berenson "Film boiling Heat Transfer from a horizontal Surface", Trans. ASME vol. $83, \mathrm{n}^{\circ} 3$, p. 351, (1951)

[38] BROMLEY NL., LEROY A. - Heat Transfer in force Convection film Boiling, Ind. Eng. Chem., 45, p. 26, (1953)

[39] SÉMÉRIA R. - Thermodynamique des Fluides diphasiques bouillants, Rev. Gen. Therm. 12, p. 211, (1973)

[40] BERLIN H. et al. - Investigation of natural Convection Film Boiling Crises, Inzh.-fiz. Zhurm, 24, n 2, p. 205, (1973)

[41] BJORNARD, GRIFFITH. - PWR Blowdown Heat Transfer, Symp. on Thermal Hydraulic Aspects of Nuclear Reactor Safety, ASME, № 27.D2, New-York, Atlanta, (1977)

[42] AHMAD SY. - Fluid to fluid Modeling of critical Heat Flux : A Compensated Distortion Model, Int. J. Heat \& Mas Transfer, vol. 16, p. 641, (1973)

[43] STEVENS GF. et al. - An experimental Investigation into Forced Convection Burn-out in Freon, with Reference to Burn-out in Water, AEEW-R 321, (1964)

[44] TONG LS. - Boiling Heat Transfer and Two-Phase Flow: John Wiley, New York, (1965)

[45] HILDEBRANDT G. - Heat Transfer to Boiling Helium under Forced Flow in a Vertical Tube, ICEC 4, Eindhoven, (1972)

[46] KUTATELADZE SS. BORISHANKII VM. - A concise Encyclopedia of Heat Transfer, Pergamon Press, (1966)

[47] CHEN Y. - Measurment of boiling Curves during Rewetting of a hot circular Duct, Int. J. Heat \& Mass Transfer, vol. 22, p. 973, (1979).

[48] GENTILE D., LLORY M. - An experimental Investigation of the boiling Crisis in a forced Convection Freon 113 Flow, ASME Winter Meeting, Miami, Nov. 1985.

[49] KATTO Y. - An Analysis of the Effect of inlet Subcooling on Critical Heat Flux of forced Convection Boiling in vertical uniformly heated Tubes, Int. J. of Heat \& Mass Transfer, vol. 22, p. 1567, (1978).

[50] SHI-CHUNB YAO - An Investigation of Transient Boiling Heat Transfer with Conjugate Nature, J. Heat Mass Transfer, vol. 10, n 6, pp. 901-909, 1983.

[51] KESSELRING, ROSCHE and BANKOFF - Transition and Film Boiling from Horizontal Strips, A.I. Ch. E., vol. 13, $n^{\circ} 4$ pp. $669-675,1967$. 